\title{
VARIACIONES INTRANUALES DEL ESPECTRO POLÍNICO DE MIEL DE NOGUEIRA DE RAMUÍN (ORENSE)
}

\author{
$M^{\text {a }}$ del Carmen SEIJO COELLO, Victoria JATO RODRIGUEZ \\ y Maa Jesús AIRA RODRÍGUEZ
}

\begin{abstract}
RESUMEN. Se ha realizado el análisis polínico de 12 muestras recogidas de forma secuencial durante el período de producción de miel en tres colmenas, ubicadas en Nogueira de Ramuín (Orense), comparando los resultados obtenidos con el espectro polínico de la mezcla final. Se observan diferencias importantes entre las muestras parciales, que ponen de manifiesto el pecoreo selectivo de las distintas colonias.
\end{abstract}

Palabras clave. Polen, miel, Orense, España.

\begin{abstract}
A palynological study of apiary's honey in twelve sequencial samples collected in Nogueira de Ramuin (Orense), during the period of honey's production, has been made. Importants differences in sequencial samples, has been observed. This fact evidence the selective sucking in differents colonies.
\end{abstract}

Key words. Pollen, honey, Orense, Spain.

\section{INTRODUCCIÓN}

La producción de miel en Galicia, se estima que puede ser del orden de 600$800 \mathrm{Tm} / \mathrm{año}$, siendo su comercialización mayoritariamente de tipo directo y local. Las características de este producto, han sido estudiadas por diversos autores tanto desde el punto de vista físico-químico (Huidobro, 1983; Huidobro et al. 1986; Serra, 1988) como palinológico (Vieitez, 1950; Sánchez et al., 1982; Terradillos, 1988; Jato et al., en prensa; Janeiro, 1990; Aira et al., 1991; entre otros).

Aunque los datos actuales no son suficientes para la tipificación de este producto (debido principalmente a la irregularidad del muestreo, ya que hay zonas importantes, cuyas mieles no han sido estudiadas todavía), al menos permiten conocer los tipos de miel más abundantes y deducir el predominio de las milflorales sobre aquellas en las que prevalece un taxon determinado.

Las mieles milflorales se producen por todo el territorio gallego, mientras que las monoflorales están ligadas a la presencia de castaños (Castanea sativa Miller), eucaliptos (Eucalyptus globulus Labill), brezo (Ericaceae) y Rubus sp.

Con el presente trabajo, se pretende contribuir a la tipificación de las mieles orensanas estudiando, además, la variación del espectro polínico a lo largo de todo el período de producción de esta miel. Con ello se podrán conocer las plantas que 
aparecen mejor representadas entre las melíferas y/o poliníferas presentes en el entorno del colmenar, determinar indirectamente la fenología de dichas plantas y observar si existe un pecoreo selectivo en las diferentes colmenas.

\section{MATERIAL Y MÉTODOS}

El colmenar que se ha utilizado para este estudio está situado en Nogueira de Ramuín (Orense) en una zona rural, en donde abundan los cultivos de huerta y viñedos. Concretamente se ubica bajo un alcornocal, en la margen izquierda del Río Miño, a $120 \mathrm{~m}$ de altitud.

La estación meteorológica más próxima (Peares) señala una precipitación media anual de $764.16 \mathrm{~mm}$, siendo de $25.14 \mathrm{~mm}$ la media de los meses en los que se realizó el muestreo.

El colmenar está formado por 14 colmenas movilistas de las que se eligieron tres para el muestreo (dos de tipo Langstroth y una Industrial).

La recogida de muestras se realizó de forma periódica, comenzando en el mes de Abril y utilizando panales, vacíos y limpios, que se colocaron en las colmenas al principio de cada mes, obteniéndose un total de 12 muestras parciales (tres por cada período). La cosecha final se recogió en el mes de Agosto y, tanto ésta como el resto de las muestras, se extrajeron por centrifugación.

Coincidiendo con la fecha de recogida de las muestras parciales, se pesaron las colmenas y los panales utilizados, para conocer la variación en la cantidad de miel o néctar. Los análisis cuantitativos, cualitativos, la cuantificación del sedimento y el índice de mielada, fueron realizados siguiendo los métodos propuestos por Louveaux et al., 1978.

\section{RESULTADOS}

\section{Representatividad de los distintos táxones en la mezcla comercial.}

La especie que alcanza mayor importancia (Tabla 1) es Castanea sativa Miller, cuyo polen se representa con un $61 \%$, acompañada de Erica arborea L. (11\%) y Rubus sp. $(10 \%)$. Otros táxones que se encuentran con menores porcentajes son: t. Erica cinerea, Eucalyptus globulus Labill, ambos con un $3 \%$ y Erica umbellata L., t. Ulex, Quercus sp., Salix sp., t. Sinapis y Daboecia cantabrica (Hudson) C. Koch que no superan el $2 \%$.

La única especie que aparece en todas las muestras analizadas es Erica arborea L., mientras que Erica australis L., que aparece incluso dominante en algún espectro parcial, no está representada en el final.

Por otra parte, existen algunas diferencias en cuanto a los tipos polínicos presentes en los espectros parciales de las distintas colmenas; así el t. Crataegus aparece en todas las muestras de la colmena L-2, pero no en las demás colmenas, lo que podría explicar su ausencia de la mezcla final (Tabla 1).

\section{Evolución del espectro polínico en las muestras de las tres colmenas.}

En la colmena Langstroth (L-1), durante el mes de Abril (Fig. 1) se observa la gran importancia de las crucíferas, en concreto del tipo Sinapis. Como acompañantes aparecen por orden de abundancia: E. australis L., t. Ulex, E. arborea L., Daboecia cantabrica (Hudson) C. Koch., Eucalyptus globulus Labill, Castanea sativa Miller y Salix sp. 


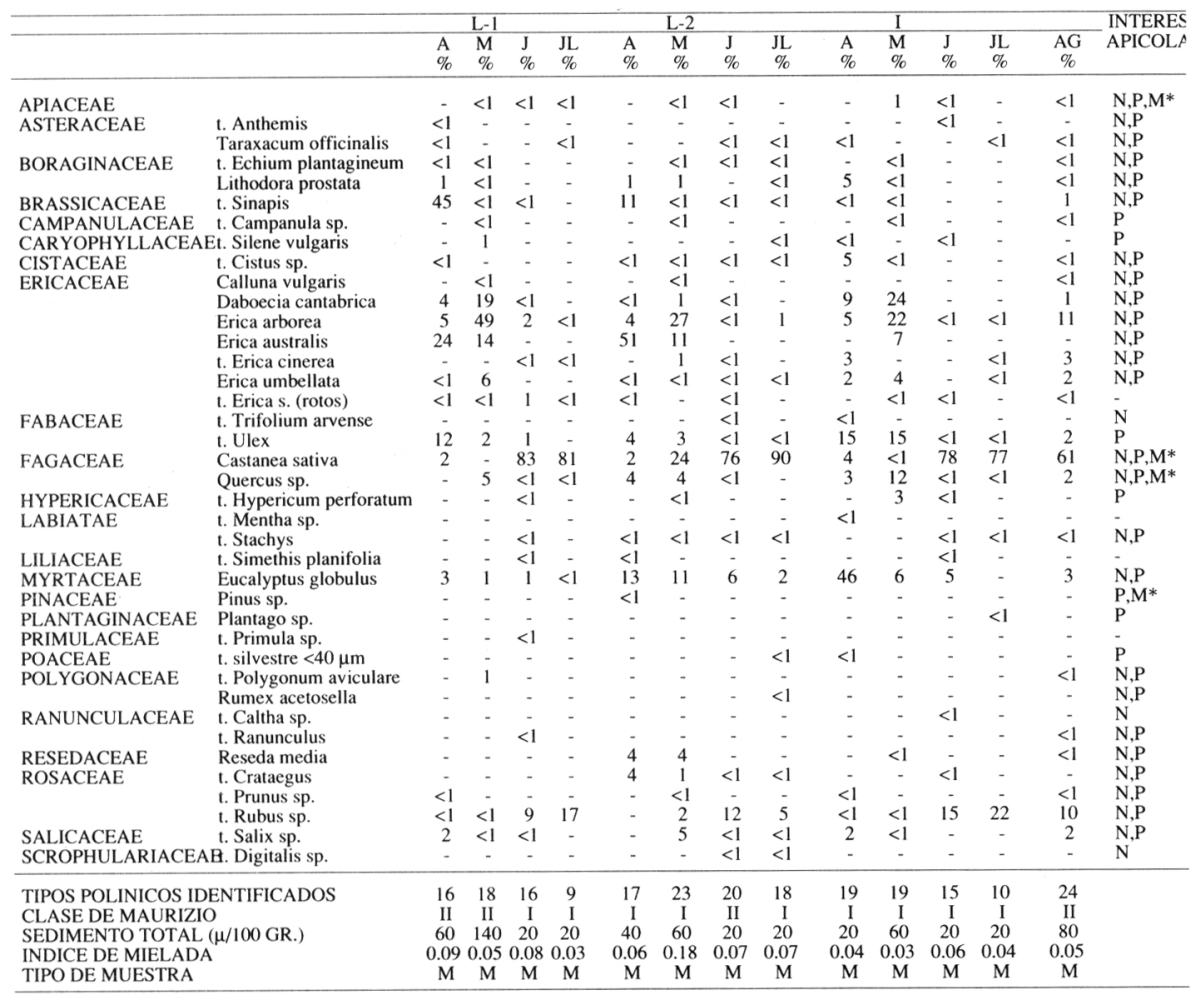

Tabla 1. Espectro polínico de la miel de Nogueira. $\mathrm{M}=$ Miel; $\mathrm{N}=$ Néctar; $\mathrm{M}^{*}=$ Mielato; $\mathrm{P}=$ Polen. A= Abril; M= Mayo; J= Junio; JL= Julio; Ag= Agosto.

Es notable la importancia de Ericaceae que se incrementa durante el mes de Mayo, en el que la suma de los porcentajes de las especies de esta familia, alcanza el $90 \%$ del total del polen de esa miel.

En las muestras correspondientes a los meses de Junio y Julio, la atractiva floración del castaño, provoca que las abejas recojan mayoritariamente néctar de dicha especie, tal como lo reflejan los espectros polínicos de ambos períodos. En Junio todavía se mantiene cierta cantidad de Erica arborea L. y aparece también como acompañante el polen de Rubus sp. que en el mes de Julio va a ser el único de importancia después del castaño.

En la otra colmena Langstroth (L-2), Erica australis L. resulta ser el taxon predominante durante el mes de Abril (Fig. 2). Como acompañantes aparecen el t. Ulex, t. Crataegus y Erica arborea L., además de algunos árboles (Quercus sp., Castanea sativa Miller y Eucalyptus globulus Labill) y algunas herbáceas (t. Sinapis y Reseda sp.).

En el mes de Mayo el espectro polínico resulta más diverso, la representación del polen de Eucalyptus globulus Labill y Quercus sp. prácticamente se mantiene, mientras que la de Castanea sativa Miller y Erica arborea L. aumenta. Rubus sp. y Salix sp. aparecen con valores escasos. 


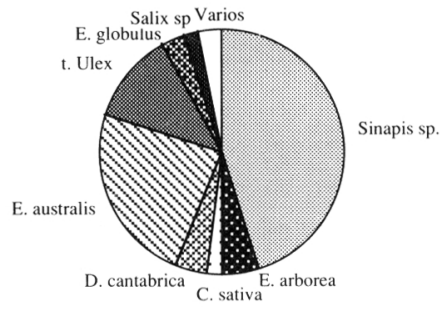

ABRIL

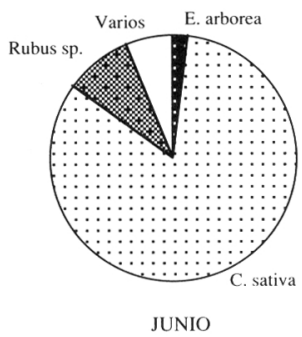

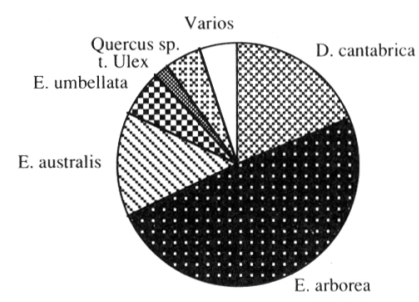

MAYO

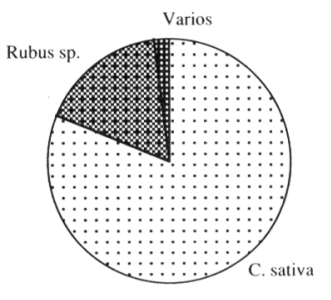

JULIO

Fig. 1. Espectro polínico de la colmena L-1 (Nogueira).

En los meses de Junio y Julio, Castanea sativa Miller, es el taxon de mayor aprovechamiento apícola, acompañado de Rubus sp. y Eucalyptus globulus Labill.

En la miel recogida en la colmena industrial (I) la especie que inicia la temporada de cosecha es Eucalyptus globulus Labill (Fig. 3).

Es importante la presencia de Ericaceae que en su conjunto alcanzan el 19\%, t. Ulex (15\%), Lithodora prostata (Loisel) Griseb (5\%), Castanea sativa Miller (4\%) y Quercus (3\%).

En Mayo, se incrementa la proporción de polen de Erica arborea L., Daboecia cantabrica (Hudson) C. Koch. y de Erica umbellata L. en menor cantidad. Por el contrario Eucalyptus globulus Labill disminuye mucho y el t. Ulex se mantiene durante este mes, desapareciendo en el siguiente.

En Junio y Julio, Castanea sativa Miller junto con Rubus sp., obtienen los mayores valores. Otra especie importante en el mes de Junio, es Eucalyptus globulus Labill.

Con respecto al número de tipos polínicos identificados en las distintas etapas, el máximo corresponde a los meses de Mayo y Junio, coincidiendo con el período de floración de la mayoría de las especies.

\section{Análisis cuantitativo, cuantificación del sedimento e índice de mielada.}

Algunas de las muestras analizadas llegan a alcanzar 85.000 granos de polen/ 10 gramos de miel, por lo que se incluyen en la clase II según Maurizio (1978) mientras que nueve de ellas pertenecen a la clase I.

En la mezcla se obtienen valores más altos desde el punto de vista cuantitativo.

Los resultados obtenidos en la cuantificación del sedimento, indican que los valores más altos, se obtienen en el mes de Mayo, en las tres colmenas muestreadas.

Finalmente, en cuanto al índice de mielada, los distintos elementos identificados, 


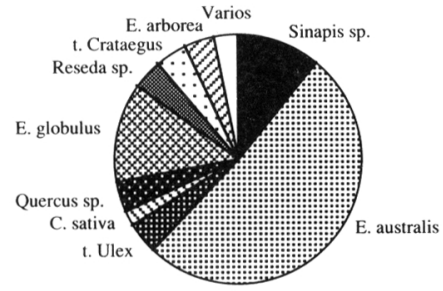

ABRIL

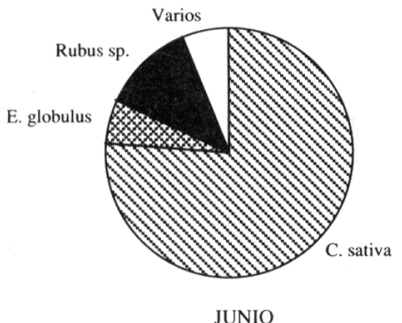

JUNIO

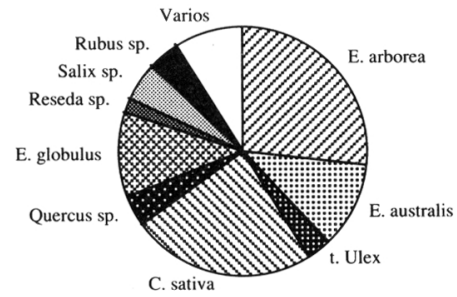

MAYO

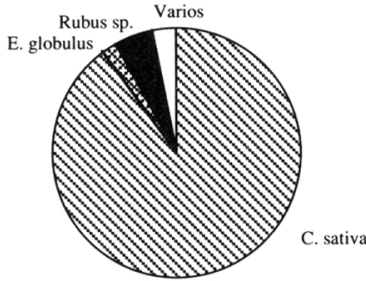

JULIO

Fig. 2. Espectro polínico de la colmena L-2 (Nogueira).

tanto de las muestras parciales como de la mezcla final, presentan valores muy bajos, por lo que se trata de una miel de flores.

\section{Evolución del peso de las colmenas.}

La cantidad de miel total recogida en el colmenar, fue de 300 kilos, lo que indica una buena producción. La colmena L-2 fue la que tuvo un menor aumento de peso desde el inicio del período de muestreo, mientras que las otras dos (L-2 e I) alcanzaron valores elevados y muy similares, apreciándose un paralelismo de estos datos con la producción de miel.

La abundante floración de Castanea sativa Miller y su importancia melífera, hace que en Junio se observe un aumento importante de peso que se mantiene durante el mes siguiente, mientras que en Agosto, la falta de néctar en el campo y la pérdida de agua por evaporación en el néctar del interior de los panales, provocan una pequeña pérdida de peso.

\section{DISCUSIÓN}

En los resultados comentados anteriormente se aprecian diferencias notables dentro del colmenar, lo que demuestra cierta selectividad de las abejas en la recogida de néctar y polen, al mismo tiempo que refleja la situación de las distintas colonias.

En el mes de Abril, ningún taxon dominante coincide en las tres colmenas muestreadas (Fig. 4) Erica australis L., t. Sinapis y Eucalyptus globulus Labill son los tipos polínicos que con frecuencias similares dominan en cada una de las tres colmenas. Erica australis L. aparece como dominante en la L-2, Sinapis en L-1 y Eucalyptus lo hace en la industrial (I).

En el mes de Mayo, se ve una clara tendencia de las abejas al pecoreo en flores de brezo, más evidente en la colmena L-1, mientras que la Industrial lo comparte con 

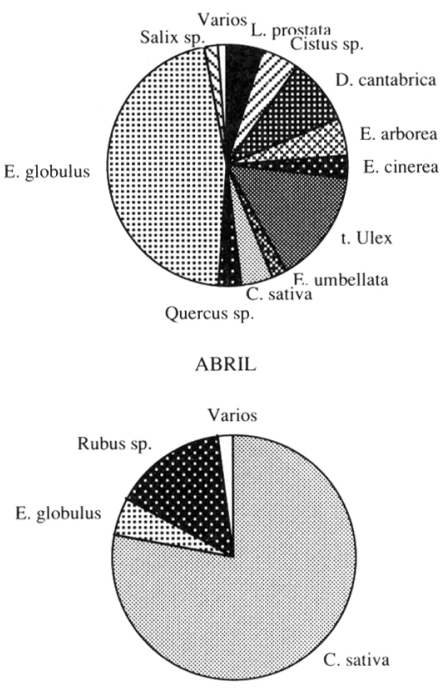

JUNIO
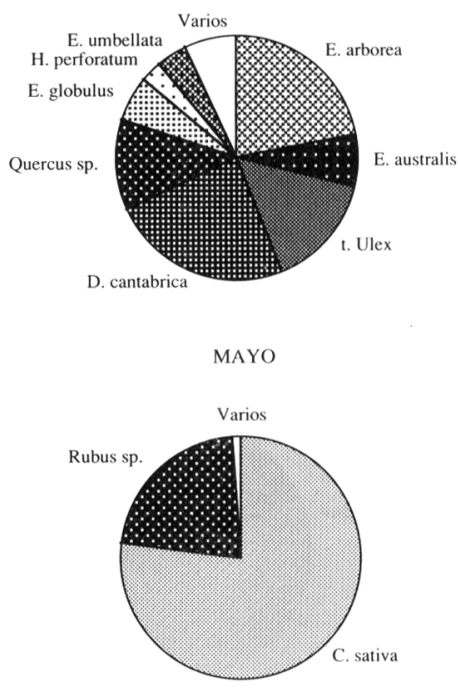

JULIO

Fig. 3. Espectro polínico de la colmena I (Nogueira).

Eucalyptus globulus Labill y otros táxones. En esta época, la colonia de L-2 descubre como fuente de néctar el castaño, lo que origina que la representación del polen de esta especie alcance el $24 \%$ frente a la ausencia en las otras colmenas.

En los meses de Junio y Julio, el gran atractivo que el castaño ofrece a las abejas, unifica de forma notable los distintos espectros, apareciendo como taxon dominante con valores similares en todas las colmenas. Las variaciones corresponden únicamente a los táxones acompañantes siendo éstos, Rubus sp. exclusivamente, o en compañía de Eucalyptus globulus Labill.

Como conclusión, para este colmenar, se podría describir una secuencia que comenzaría con la floración de t. Sinapis (cuya importancia melífera ha sido señalada por Janeiro, 1990 en otros trabajos sobre mieles gallegas) y Eucalyptus globulus Labill, seguidamente aparecen las Ericaceae muy importantes tanto por su valor en la producción de miel, dada la calidad de su néctar (Sánchez et al., 1989), como por la gran distribución y abundancia en el monte gallego. Finalmente, la floración de Castanea sativa Miller muy abundante en el emplazamiento del colmenar, junto con Rubus sp., acapara la recolección de las abejas en los meses estivales.

A pesar de la importancia de estos táxones para la producción de miel, no todas las colonias presentan la misma tendencia en el pecoreo, tal como se puede deducir de los análisis polínicos cualitativos de las diferentes colonias. Este fenómeno se puede explicar mediante un modelo de pecoreo selectivo, ya que en ausencia de una abundante secreción nectarífera, las colonias tienden a especializarse en la recolección de un tipo de néctar.

Por otra parte, la abundancia de un taxon determinado, no garantiza su presencia en la miel, tal como ocurre con Quercus sp., cuyo polen aparece con valores muy bajos, como corresponde a especies de carácter anemófilo. Por otra parte, su importancia como productora de mielatos (Meixide, 1978; Andreev, 1976), tampoco se manifiesta en la miel analizada. 

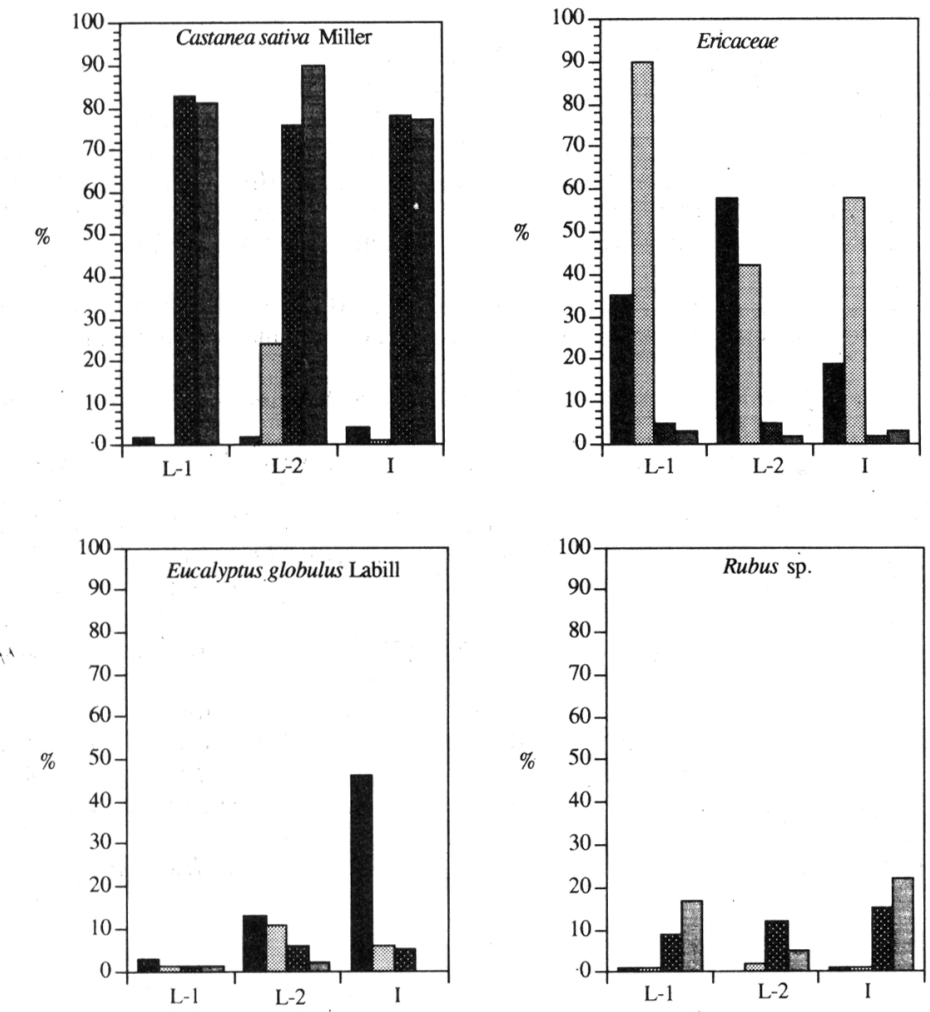

ABRIL

MAYO

JUNIO 國 JULIO

Fig. 4. Evolución de los táxones más importantes (Nogueira).

Se observa una relación positiva entre la fenología de las plantas melíferas y la presencia de su polen en las muestras analizadas; en concreto con respecto a los brezos, Erica australis L. y Erica arborea L. son las primeras especies en florecer y se identifican en las muestras de Abril y Mayo. A continuación florece Erica umbellata L. y en el mes de Junio Daboecia cantabrica (Hudson) C. Koch y las especies incluídas en el t. Erica cinerea, que aparecen en los espectros correspondientes a dicha época.

Finalmente en cuanto a la caracterización de esta miel, por su orígen floral, se incluiría entre las milflorales con altos porcentajes de polen de castaño $(61 \%)$ ya que dichos valores no son suficientes para determinar su monofloralidad.

\section{BIBLIOGRAFÍA}

AIRA RODRÍGUEZ, M.J., P. RAMIL REGO Y M.P. SAÁ OTERO -1991- Identificación polínica de Ericaceae en mieles gallegas. Acta Botanica Malacitana, 15: 27-32. 
ANDREEV,P.G. -1976- Recolección del mielato por las abejas de varias razas en las condiciones de la cuenca del Volga. Ed. Apimondia, 182-184.

HUIDOBRO CANALES, J.F. -1983- La miel. Algunos parámetros de interés de su control de calidad. Tesis Doctoral. Facultad de Farmacia. Universidad de Santiago.

HUIDOBRO CANALES, J.F., J. SIMAL Y L.A. TERRADILLOS -1986- Significado de los principales parámetros de la miel, valor alimenticio y usos. Boletín Apícola, 57-68.

JANEIRO CARAMES, B. -1990- Correlación entre la fenología de la flora melífera y el espectro polínico de dos mieles gallegas. Memoria de Licenciatura. Facultad de Biología. Universidad de Santiago.

JATO RODRIGUEZ, V., A. SALA LLINARES, I. IGLESIAS FERNÁNDEZ \& M. SUAREZ CERVERA -en prensa-Pollen characterization of Orense's honeys (NW Spain). Jour. of Apic. Research.

LOUVEAUX, J., A. MAURIZIO \& G. VORWOHL -1978- Methods of Melissopalynology. Bee World, 59(4): 139-157.

MAURIZIO, A. -1978- Weitere untersuchungen au pollenhöschen Verlay H.R. Sanerländer \& Co., Aasan.

MEIXIDE VILARIÑO, J.L. -1978- Contribución al estudio de los áfidos productores de mielato en Galicia. Su aprovechamiento por la abeja melífera. Boletín Apícola, 24: 25-33.

SÁNCHEZ CUNQUEIRO, C. Y C. SAENZ LAÍN -1982- Análisis polínico de mieles de Pontevedra (España). Lazaroa, 4: 253-268.

SÁNCHEZ SÁNCHEZ , J. Y X. GIRALDEZ FERNÁNDEZ -1989- Brezales salmantinos. Vida Apícola, 33: 33-34.

SERRA BONHEVI, J. -1988- Propietés physico-chimiques, composition et spectre pollinique des miels de Lavandula latifolia Med. produits en Espagne. Sciences des Aliments, 8: 295-307.

TERRADILLOS, L.A. -1988- Estudio microscópico del sedimento de la miel y su aplicación en la caracterización de mieles de Galicia. Tesis Doctoral. Facultad de Farmacia. Universidad de Santiago.

VIEITEZ CORTIZO, E. -1950- El polen de las mieles de Galicia. Anales de Edaf. y F. Veg., X: 79-99.

(Aceptado para su publicación en Julio de 1991)

Dirección de las autoras: M.C. SEIJÓ COELLO y V. JATO RODRÍGUEZ: Departamento de Recursos Naturales y Medio Ambiente. Esc. Universitaria de E.G.B. Universidad de Vigo. 32001. Orense. M.J. AIRA RODRIGUEZ: Departamento de Biología Vegetal. Facultad de Farmacia. Universidad de Santiago. 15706. Santiago (La Coruña). 